\title{
Comparison of the Clinical Performances of the Immunoenzymometric Assays for N-Terminal and C-Terminal Type I Collagen Telopeptides and the HPLC Assay for Pyridinium Cross-Links
}

\author{
Paolo Bettica ${ }^{1}$, Marco Masino ${ }^{2}$, Enrico Cucinotta ${ }^{2}$, Tarcisio Vago ${ }^{1}$, Guido Norbiato ${ }^{1}$, Luigi Moro ${ }^{3}$, \\ Karem Noris Suarez ${ }^{3}$, Milena Romanello ${ }^{3}$ and Maurizio Bevilacqua ${ }^{1}$ \\ 1 Servizio di Endocrinologia, Ospedale "L. Sacco", Milano, Italy \\ 2 Assay Laboratory, USSL 44, Voghera, Italy \\ ${ }^{3}$ Dipartimento di Biochimica, Biofisica e Chimica delle Macromolecole, Universitá degli Studi di Trieste, \\ Trieste, Italy
}

Summary: We evaluated the clinical performances of the immunoenzymometric assays for type I collagen $\mathrm{N}$ terminal and C-terminal telopeptides and the HPLC assay for total deoxypyridinoline, in distinguishing between subjects with a moderately increased bone resorption rate (women in postmenopause) and subjects with normal bone resorption rate (women in premenopause). The postmenopausal group consisted of 61 women who had been in menopause for no more than 10 years, while the premenopausal group consisted of 52 healthy women with normal menstrual cycles. The biochemical markers were measured in a 24 hour urine sample and the results expressed as the molar ratio with urinary creatinine. The clinical performances were estimated by calculating the accuracy (as the area under a Receiver Operated Characteristic (ROC) curve: mean \pm SEM) and the discriminating power (as score) of each assay in distinguishing postmenopausal subjects from premenopausal subjects. Type I collagen C-terminal telopeptide, type I collagen $\mathrm{N}$-terminal telopeptide and total deoxypyridinoline were significantly higher in the postmenopausal than in the premenopausal group $(\mathrm{p}<0.001)$. Accuracies of these three markers ranged from $66.8 \pm 5.1 \%$ to $76.8 \pm 4.3 \%$, while $\mathrm{Z}$ scores ranged from 3.54 to 5.67 . Type I collagen C-terminal telopeptide, type I collagen $\mathrm{N}$-terminal telopeptide and total deoxypyridinoline were not significantly different in their accuracy or discriminating power. All markers were highly correlated with coefficients of correlation ranging from 0.61 to 0.77 .

In summary, this study shows that

1) the immunoenzymometric assays for type I collagen N-terminal telopeptide and type I collagen C-terminal telopeptide show a high accuracy and disciminating power in distinguishing subjects with different bone resorption rate;

2) the results obtained with these immunoenzymometric assays are comparable to those obtained with the HPLC assay for total deoxypyridinoline.

In conclusion our data support the use of the immunoenzymometric assays for type I collagen $\mathrm{N}$-terminal telopeptide and type I collagen C-terminal telopeptide for estimating bone resorption.

\section{Introduction}

The use of biochemical markers of bone resorption seems increasingly important in the diagnosis and follow-up of metabolic bone diseases, particularly osteoporosis. In a previous paper Bettica and colleagues (1) have shown that hydroxyproline, the time-honoured biochemical marker of bone resorption, is adequate for distinguishing subjects with large increases in bone resorption rate (Pagetics and children) from normal subjects, but has limited use in estimating small differences in bone resorption rate (postmenopausal osteoporosis). On the other hand, galactosyl hydroxylysine and the pyri- dinium cross-links were shown to be relatively efficient in estimating both small and big differences in bone resorption rate $(1,2)$.

Unfortunately these assays, particularly the pyridinium cross-links, are time-consuming (3), and an immunometric assay is desirable. In recent months, different immunoenzymometric assays that measure type I collagen telopeptides (both $\mathrm{C}$ - and $\mathrm{N}$-terminal) have been proposed $(4,5)$, and preliminary data on these assays in different metabolic bone diseases have shown promising results $(4,5)$. However, to the best of our knowledge, the clinical performances of these new assays have not yet been 
compared in the same subjects, in order to establish their respective accuracies in discriminating different rates of bone resorption.

This study sought to evaluate the clinical performances of these new immunoenzymometric assays in comparison with those of the HPLC assay for total deoxypyridinoline, and to estimate the relationships among these markers.

\section{Patients and Methods}

Two groups of subjects were selected for the study. The control group (premenopausal) consisted of 52 women with regular menstrual cycle and regular ovulation (as demonstrated by a serum progesterone level $>5 \mu \mathrm{g} / \mathrm{l}$ around the 20th day of cycle) with an age of $34.2 \pm 5.8$ years (mean \pm SD), recruited among the hospital personnel; none of them had any disease or was taking any medication known to affect bone turnover. Their weight was $62.2 \pm 8.1$ $\mathrm{kg}$ and their height $160.3 \pm 11 \mathrm{~cm}$. The second group (postmenopausal) consisted of 61 women in postmenopause ( $<10$ years; mean $7.1 \pm 3.7$ years) with an age of $57.3 \pm 3.5$ years, who attended our Endocrinology Outpatient Clinic. Their weight was $61.4 \pm 8.3 \mathrm{~kg}$ and their height $159.4 \pm 5.5 \mathrm{~cm}$. The bone mineral density of the spine of the postmenopausal group (measured by dual X-ray absorptiometry in the anteroposterior projection with a QDR2000 bone densitometer, Hologic) was $0.821 \pm 0.071 \mathrm{~g} / \mathrm{cm}^{2}$, corresponding to a $t$ score of $-2.31 \pm 0.66$ (calculated using the normal young female caucasian database provided by Hologic). None of the subjects in the postmenopausal group was taking any medication known to affect bone turnover.

All subjects collected a 24 hour urine sample and aliquots were stored at $-20^{\circ} \mathrm{C}$ until use. Total deoxypyridinoline, type I collagen $\mathrm{N}$-terminal telopeptide, type I collagen C-terminal telopeptide and creatinine were measured. All samples were measured in the same run to avoid inter-assay variation.

Total deoxypyridinoline was measured by HPLC with a commercial kit (Chrom-Links in HPLC, BIORAD). Type I collagen Nterminal telopeptide was measured with a commercial kit with a monoclonal antibody specific for the cross-linked type I collagen $\mathrm{N}$-terminal telopeptide (Osteomark, Ostex, USA). Type I collagen C-terminal telopeptide was measured with a commercial kit (Crosslaps ELISA, Osteometer, Denmark) with a polyclonal antibody raised against a synthetic sequence of type I collagen C-terminal telopeptide. Creatinine was measured by an automatic assay based on the Jaffe method.

\section{Statistics}

The clinical performances of the different markers were estimated by calculating the accuracy and the discriminating power a distinguishing postmenopausal from premenopausal women (1). The accuracy was calculated as the mean \pm SEM area under a receiver operated characteristic (ROC) curve that was drawn by calculating the sensitivity and the false positive ratio at nine different threshold values (6). The method used to select the threshold values was the same for each assay. The values selected ranged from mean $-2 \mathrm{SD}$ to mean $+2 \mathrm{SD}$ of the control subjects. The discriminating power was calculated as the $\mathrm{Z}$ score with the aid of the following formula (7):

$$
\mathrm{Z} \text { score }=\frac{\text { (postmenopausal mean }- \text { premenopausal mean })}{\sqrt{\left[\frac{(\text { postmenopausal SD })^{2}}{\text { postmenopausal } n}+\frac{(\text { premenopausal SD })^{2}}{\text { premenopausal } n}\right]}}
$$

The accuracies of the different tests were compared by the method of Hanley \& McNeil (6). The relationships among the different markers were estimated by linear regression analysis.
Finally, to test the agreement among total deoxypyridinoline, type I collagen N-terminal telopeptide and type I collagen Cterminal telopeptide, we calculated for each postmenopausal woman and for each marker a $t$ score ( $t$ score $=$ (postmenopausal subject value - premenopausal mean value)/premenopausal standard deviation) and for each pair of markers we estimated the correlation between the mean $t$ score (independent variable) and the difference between the $\mathrm{t}$ scores (dependent variable) by the method of Bland \& Altman (8).

\section{Results}

As shown in figure 1 the urinary excretion of total deoxypyridinoline/creatinine, type I collagen $\mathrm{N}$-terminal telopeptide/creatinine and type I collagen C-terminal telopeptide/creatinine was significantly higher in postmenopausal women. The mean increase was $32 \%$ for total deoxypyridinoline/creatinine, $31 \%$ for type I collagen $\mathrm{N}$-terminal telopeptide/creatinine and $65 \%$ for type I collagen C-terminal telopeptide/creatinine.

Table 1 shows the clinical performances of the three markers. The accuracies of total deoxypyridinoline/ creatinine, type I collagen $\mathrm{N}$-terminal telopeptide/creatinine and type I collagen C-terminal telopeptide/creatinine were comparable and ranged from $66.8 \pm 5.1 \%$ (type I collagen $\mathrm{N}$-terminal telopeptide/creatinine) to $76.8 \pm 5.3 \%$ (type I collagen C-terminal telopeptide/ creatinine). The discriminate powers ranged from 3.54 (type I collagen $\mathrm{N}$-terminal telopeptide/creatinine) to 5.67 (type I collagen C-terminal telopeptide/creatinine). The accuracies and discriminating powers were not significantly different for each of the three markers.

As shown in table 2 all the markers were highly correlated with coefficients of correlation ranging from 0.61 (total deoxypyridinoline vs type I collagen Cterminal telopeptide) to 0.77 (total deoxypyridinoline vs type I collagen N-terminal telopeptide) when the absolute values were considered (tab. 2a), and from 0.502 (total deoxypyridinoline/creatinine vs type I collagen C-terminal telopeptide/creatinine) to 0.681 (type I collagen $\mathrm{N}$-terminal telopeptide/creatinine vs type I collagen C-terminal telopeptide/creatinine) when creatinine ratios were used (tab. 2b). As shown in figure 2 there was also a good agreement among the three markers, since none of the slopes of the linear regressions, calculated according to the method of Bland \& Altman (8), was significantly different from zero. Good agreement between the three markers is also indicated by the fact that there was no improvement in accuracy in distinguishing premenopausal and postmenopausal women when total deoxypyridinoline, type I collagen $\mathrm{N}$-terminal telopeptide and type I collagen C-terminal telopeptide were used in series (i.e. a result is positive when both test results are above the threshold value) or in parallel (i.e. a result is positive when at least one test result is above the threshold value) (tab. 3). 

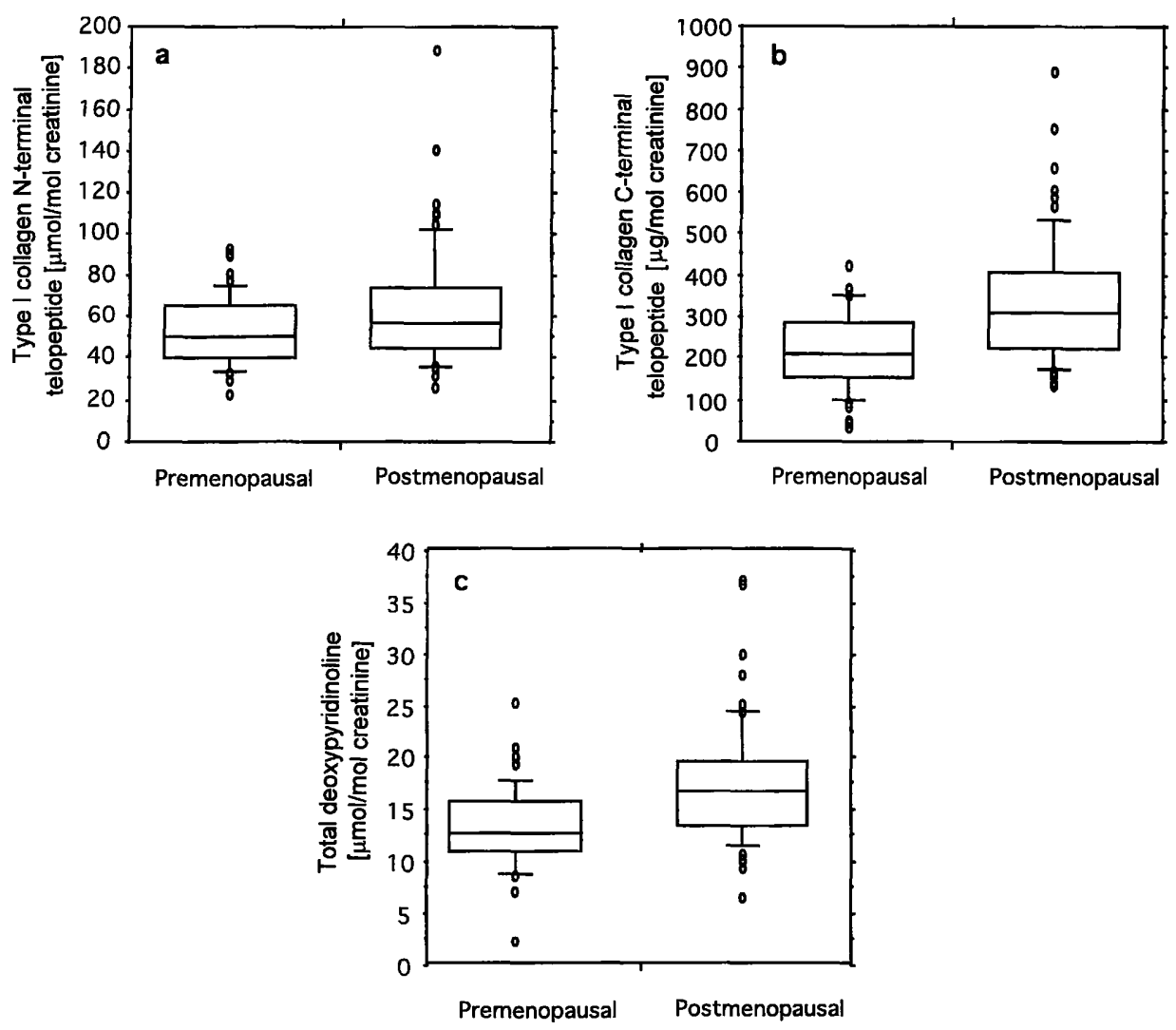

Fig. 1 Urinary excretion of

a) type I collagen $\mathrm{N}$-terminal telopeptide/creatinine,

b) type I collagen C-terminal telopeptide/creatinine in premenopausal and postmenopausal women and

c) total deoxypyridinoline/creatinine.

\section{Discussion}

When measured in urine, a biochemical marker of bone resorption ideally has the four following properties.

1) Bone specificity.

2) Specificity for bone resorption.

3) Absence of metabolism and clearance from the body only by the kidney.

4) Specificity, sensitivity, reproducibility and simplicity of the assay.

A marker with such characteristics would be highly accurate in distinguishing subjects with different bone resorption rates. Hydroxyproline is neither specific for bone $(9-12)$ nor for bone resorption (10), moreover it is mostly metabolised before being excreted in urine (13). As a consequence hydroxyproline is a poor bone resorption marker (1). In the search of specific markers of bone resorption, different molecules have been proposed in recent years. In particular, great interest has been shown in the study of pyridinium crosslinks and of type I collagen telopeptides. Regarding pyridinium cross-links, pyridinoline is present in many different tissues (14) and deoxypyridinoline has been
Results are mean \pm SD. For all three markers postmenopausal women showed a significantly larger excretion than premenopausal women $(\mathrm{p}<0.001)$.

detected in bone, dentine, aorta and ligaments (15). Both molecules are formed during collagen maturation, and are therefore released from tissues only following collagen degradation. Nothing is known about the presence or absence of metabolism, or the extent of renal excretion of these molecules. In particular, since urinary pyridinium cross-links are $40 \%$ free and $60 \%$ peptide-bound (16), we do not know whether they are released from bone in this proportion, or whether they become free due to the action of circulating or renal peptidases. Total pyridinium cross-links are measured by HPLC with an assay that is specific

Tab. 1 Accuracies (mean \pm SEM) and discriminating powers $(Z$ score) of total deoxypyridinoline/creatinine, type I collagen N-terminal telopeptide/creatinine and type I collagen C-terminal telopeptide/creatinine in distinguishing postmenopausal subjects from premenopausal subjects.

\begin{tabular}{|c|c|c|c|c|c|}
\hline \multicolumn{2}{|c|}{$\begin{array}{l}\text { Total deoxy- } \\
\text { pyridinoline/ } \\
\text { creatinine }\end{array}$} & \multicolumn{2}{|c|}{$\begin{array}{l}\text { Type I collagen } \\
\text { N-terminal } \\
\text { telopeptide/ } \\
\text { creatinine }\end{array}$} & \multicolumn{2}{|c|}{$\begin{array}{l}\text { Type I collagen } \\
\text { C-terminal } \\
\text { telopeptide/ } \\
\text { creatinine }\end{array}$} \\
\hline Accuracy & $Z$ score & Accuracy & $Z$ score & Accuracy & Z score \\
\hline $70.8 \pm 4.9$ & 4.21 & $66.8 \pm 5.1$ & 3.54 & $76.8 \pm 4.3$ & 5.67 \\
\hline
\end{tabular}


Tab. 2a Coefficients of correlation ( $r$ ) among absolute values of total deoxypyridinoline, type I collagen $\mathrm{N}$-terminal telopeptide and type I collagen C-terminal telopeptide. Data are expressed as $\mathrm{nmol} / \mathrm{l}$ (total deoxypyridinoline and Type I collagen $\mathrm{N}$-terminal telopeptide) or $\mu \mathrm{g} / 1$ (Type I collagen C-terminal telopeptide). All coefficients are highly significant $(\mathrm{p}<0.001, \mathrm{n}=113)$.

\begin{tabular}{lll}
\hline & $\begin{array}{l}\text { Total deoxy- } \\
\text { pyridinoline }\end{array}$ & $\begin{array}{l}\text { Type I collagen } \\
\text { C-terminal } \\
\text { telopeptide }\end{array}$ \\
\hline $\begin{array}{l}\text { Type I collagen } \\
\text { N-terminal telopeptide }\end{array}$ & 0.775 & 0.713 \\
$\begin{array}{l}\text { Type I collagen } \\
\text { C-terminal telopeptide }\end{array}$ & 0.612 & - \\
\hline
\end{tabular}

Tab. 2b Coefficients of correlation (r) among total deoxypyridinoline/creatinine, type I collagen $\mathrm{N}$-terminal telopeptide/creatinine and type I collagen C-terminal telopeptide/creatinine. Data are expressed as $\mu \mathrm{mol} / \mathrm{mol}$ of creatinine (total deoxypyridinoline and type I collagen $\mathrm{N}$-terminal telopeptide) or $\mu \mathrm{g} / \mathrm{mol}$ of creatinine (type I collagen $\mathrm{C}$-terminal telopeptide). All coefficients are highly significant $(\mathrm{p}<0.0001, \mathrm{n}=113)$.

\begin{tabular}{lll}
\hline & $\begin{array}{l}\text { Total deoxy- } \\
\text { pyridinoline/ } \\
\text { creatinine }\end{array}$ & $\begin{array}{l}\text { Type I collagen } \\
\text { C-terminal } \\
\text { telopeptide/ } \\
\text { creatinine }\end{array}$ \\
\hline $\begin{array}{c}\text { Type I collagen N-terminal } \\
\text { telopeptide/creatinine }\end{array}$ & 0.549 & 0.681 \\
$\begin{array}{c}\text { Type I collagen C-terminal } \\
\text { telopeptide/creatinine }\end{array}$ & 0.502 & - \\
\hline
\end{tabular}

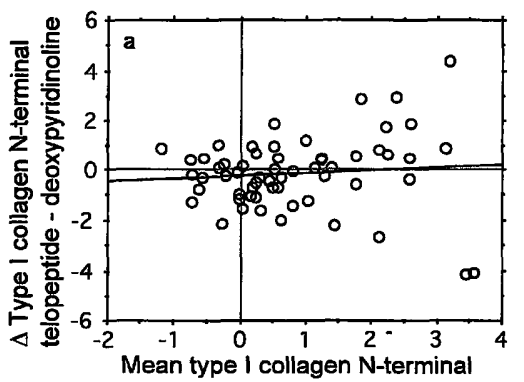

and sensitive, but time-consuming (3). Type I collagen telopeptides are present in all tissues that contain type I collagen $(4,5)$, the largest portion being bone. The method used to measure type I collagen $\mathrm{N}$-terminal telopeptide uses the cross-linked telopeptide as the antigen (4); therefore the measured type I collagen $\mathrm{N}$ terminal telopeptides arise from mature collagen degradation. The method used to measure type I collagen C-terminal telopeptide uses, as the antigen, a synthetic peptide corresponding to eight amino acids of the Cterminal telopeptide of the $\alpha_{1}$ chain of type I collagen, and it does not contain a pyridinium cross-link (5); therefore the possibility of measuring peptides that derive from collagen that was degraded before deposition cannot be excluded a priori. Very little is known about the fate of the telopeptides after their release from tissues. In this regard, a recent study has shown that type I collagen $\mathrm{N}$-terminal telopeptide is released as such from bone resorbed by osteoclasts (17). Type I collagen $\mathrm{N}$-terminal telopeptide and type I collagen $\mathrm{C}$ terminal telopeptide are measured by immunoenzymometric assays, which are specific, sensitive, fairly reproducible and simple $(4,5)$. Altogether these data show that total deoxypyridinoline, type I collagen $\mathrm{N}$ terminal telopeptide and type I collagen C-terminal telopeptide fulfil part of the requirements for an ideal bone resorption marker. In this study we evaluated whether these tests were able to distinguish different rates of bone resorption. For this purpose, we eval-

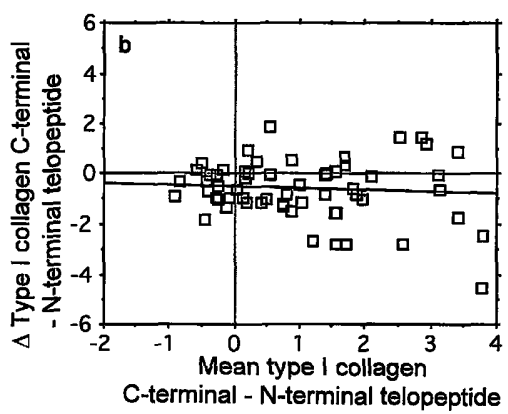

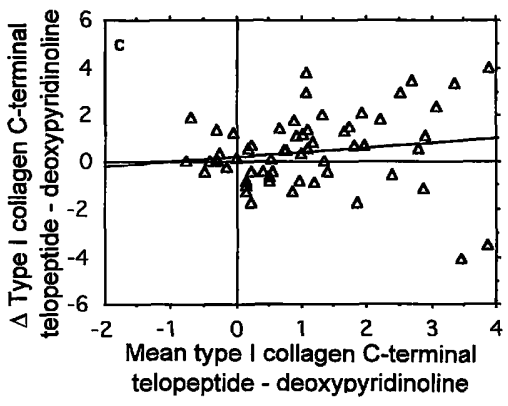

Fig. 2 Linear regression between the difference $\Delta$ of the $t$ score and the mean $t$ score calculated in postmenopausal women for total deoxypyridinoline/creatinine, type I collagen $\mathrm{N}$-terminal telopeptide/creatinine and type I collagen C-terminal telopeptide/creatinine, $(n=61)$, a: type I collagen $\mathrm{N}$-terminal telopeptide/creatinine - total deoxypyridinoline/creatinine: $\mathrm{r}=0.08, \mathrm{p}=0.52$;

b: type I collagen N-terminal telopeptide/creatinine - type I collagen C-terminal telopeptide/creatinine: $\mathrm{r}=-0.07, \mathrm{p}=0.58$; c: total deoxypyridinoline/creatinine - type I collagen $C$-terminal telopeptide/creatinine: $\mathrm{r}=0.15, \mathrm{p}=0.26$ ). 
Tab. 3 Accuracies (mean \pm SEM), in distinguishing premenopausal and postmenopausal women, of the combined use of total deoxypyridinoline/creatinine, type I collagen N-terminal telopeptide/creatinine and type I collagen C-terminal telopeptide/creatjnine.

\begin{tabular}{lll}
\hline & In series & In parallel \\
\hline $\begin{array}{l}\text { Type I collagen N-terminal } \\
\text { telopeptide/creatinine } \\
\quad+\text { type I collagen C-terminal } \\
\text { telopeptide/creatinine }\end{array}$ & $49.1 \pm 5.5$ & $69.4 \pm 4.9$ \\
$\begin{array}{l}\text { Type I collagen N-terminal } \\
\text { telopeptide/creatinine }+ \text { total } \\
\text { deoxypyridinoline/creatinine }\end{array}$ & $48.7 \pm 5.5$ & $69.7 \pm 4.9$ \\
$\begin{array}{l}\text { Type I collagen C-terminal } \\
\text { telopeptide/creatinine }+ \text { total } \\
\text { deoxypyridinoline/creatinine }\end{array}$ & $51.2 \pm 5.5$ & $74.5 \pm 4.6$ \\
\hline
\end{tabular}

uated the accuracy and the discriminating power of the tests in distinguishing a group of women in early postmenopause, i. e. a group of subjects with a moderate increase in bone resorption rate, from a group of premenopausal women, i.e. a group of subjects with a normal bone resorption rate.

Accuracy defines how often a test is able to place correctly a subject in the group he or she belongs to for a certain characteristic, in our case the bone resorption rate (18). The major factors that influence the estimate of the accuracy of a test are the "true disease status" (in our case the moderately increased bone resorption rate in the postmenopausal group) and the decision biases (19). Regarding the "true disease status", a moderate increase in bone resorption rate has been shown for women after menopause (20), which in turn determines an increase in the urinary excretion of the markers tested in this study $(5,21-$ 23). Regarding the decision biases, the receiver operated characteristic (ROC) curve analysis, that estimates the accuracy of a test using different threshold values, avoids such an interfering factor (19). Type I collagen $\mathrm{C}$-terminal telopeptide, type I collagen $\mathrm{N}$-terminal telopeptide and total deoxypyridinoline showed a comparatively high accuracy in placing the premenopausal subjects in the group with normal bone resorption rate and the postmenopausal subjects in the group with a moderately increased bone resorption rate. The accuracies ranged from $66.8 \pm 5.1 \%$ for type I collagen Nterminal telopeptide/creatinine to $76.8 \pm 4.3 \%$ for type I collagen C-terminal telopeptide/creatinine, and were not significantly different.

The discriminating power, on the other hand, defines how well a test distinguishes two groups of subjects that differ for a certain characteristic, which in our case was again the bone resorption rate. The test with the highest discriminating power will be the test that shows the highest difference between the mean of the two groups with the lowest dispersion. A $\mathrm{Z}$ score higher than 3 defines a high discriminating power. The differences between the mean of the premenopausal and postmenopausal groups were not similar for total deoxypyridinoline/creatinine, type I collagen $\mathrm{N}$-terminal telopeptide/creatinine and type I collagen C-terminal telopeptide/creatinine, ranging from $31 \%$ for type I collagen N-terminal telopeptide/ creatinine to $65 \%$ for type I collagen C-terminal telopeptide/creatinine. However, the estimated discriminating powers were not significantly different, suggesting that the tests that show the biggest difference between the mean of the two groups have also a bigger dispersion around the mean. Since all the assays employed in this study have similar coefficients of variation $(\mathrm{CV}<10 \%)$, these differences in variability might rather be due to the biological variance of the markers.

The secondary aim of this study was to evaluate the relationships among the markers tested. To do so, we used both the urinary concentrations of the markers and the creatinine ratio, to ensure that the relationships among the markers were not influenced by their relationship with creatinine excretion and by sample dilution. All markers were highly correlated both when absolute values and creatinine ratios were used, confirming previous publications $(4,5,21,24,25)$.

Finally, we evaluated the extent of agreement among the results obtained with the three different tests. For this purpose, we used the method of Bland \& Altman (8) which evaluates the agreement of two tests by calculating the linear regression between the mean of the two tests (independent variable) and the difference of the two tests (dependent variable). A non-significant regression shows that the two tests analysed are in good agreement. To normalize the results obtained with total deoxypyridinoline, type I collagen N-terminal telopeptide and type I collagen C-terminal telopeptide, we calculated for each assay and each postmenopausal woman a $t$ score, and we used the mean and the difference of the $t$ scores in the linear regression. The calculated linear regression (8) showed a non-significant slope for all markers, demonstrating that the results obtained with type I collagen $\mathrm{N}$-terminal telopeptide, type I collagen C-terminal telopeptide and total deoxypyridinoline are in good agreement.

In conclusion we showed that the new immunoenzymometric assays that measure $\mathrm{N}$-terminal and $\mathrm{C}$-terminal telopeptides of type I collagen have the same accuracy and discriminating power of the HPLC assay for total deoxypyridinoline in distinguishing subjects with moderately increased bone resorption rate from normal subjects. Our data on the relationship among these three 
markers show that these markers are correlated and in good agreement, thus supporting the use of all these as-

\section{References}

1. Bettica P, Moro L, Robins S, Taylor AK, Talbot J, Singer F, Baylink DJ. Bone resorption markers: galactosyl hydroxylysine, pyridinium crosslinks, hydroxyproline compared. Clin Chem 1992; 38:2313-8.

2. Bettica P, Taylor AK, Talbot J, Moro L, Talamini R, Baylink J. Clinical performances of galactosyl hydroxylysine, pyridinoline and deoxypyridinoline in postmenopausal osteoporosis. J Clin Endocrinol Metab 1996; 81:542-6.

3. Bettica P, Baylink DJ, Moro L. Galactosyl hydroxylysine and deoxypyridinoline: a methodological comparison. Eur $\mathrm{J}$ Clin Chem Clin Biochem 1993; 31:459-65.

4. Hanson DA, Weis MAE, Bollen AM, Maslan SL, Singer FR, Eyre DR. A specific immunoassay for monitoring human bone resorption: quantitation of type I collagen cross-linked N-telopeptides in urine. J Bone Miner Res 1992; 7:1251-8.

5. Garnero P, Ginetys E, Riou JP, Delmas PD. Assessment of bone resorption with a new marker of collagen dagradation in patients with metabolic bone disease. J Clin Endocrinol Metab 1994 a; 79:780-5.

6. Hanley JAM, McNeil BJ. A method of comparing the areas under receiver operated characteristic curves derived from the same cases. Radiology 1983; 148:839-43.

7. Armitage P. Statistica Medica. Milano: Feltrinelli, 1975:122.

8. Bland JM, Altman DG. Statistical methods for assessing agreement between two methods of clinical measurement. Lancet 1986; i:307-10.

9. Nimni ME. Collagen: its structure and function in normal and pathological connective tissues. Semin Arthritis Rheum 1974; $4: 95-150$.

10. Robins SP. Turnover of collagen. In: Weiss J, Jayson MIV, editors. Collagen in health and disease. London: Churchill Livingstone, 1982:160-78.

11. Kivirikko KI. Urinary excretion of hydroxyproline in health and disease. Int Rev Connect Tissue Res 1970; 5:93-163.

12. Reid KBM. A collagen-like amino acid sequence in a polypeptide chain of human Clq (a subcomponent of the first component of complement). Biochem J 1974; 141:189-203.

13. Weiss $\mathrm{PH}$, Klein $\mathrm{L}$. The quantitative relationship of urinary peptide hydroxyproline excretion to collagen degradation. $\mathrm{J}$ Clin Invest 1969; 48:1-10.

14. Eyre DR, Koob TJ, Van Ness KP. Quantition of hydroxypyridinium crosslinks in collagen by high-performance liquid chromatography. Anal Biochem 1984; 137:380-8.

15. Seibel MJ, Robins SP, Bilezikian JP. Urinary pyridinium crosslinks of collagen: specific markers of bone resorption says for estimting bone resorption in the diagnosis and follow-up of osteoporosis.

in metabolic bone disease. Trends Endocrinol Metab 1992; $3: 263-70$.

16. Seyedin SM, Kung VT, Daniloff YN, Hesley RP, Gomez B, Nielsen LA, et al. Immunoassay for urinary pyridinoline: the new marker of bone resorption. J Bone Miner Res 1993; $8: 635-41$.

17. Apone S, Fevold K, Lee M, Eyre D. A rapid method for quantifying osteoclast activity in vitro. J Bone Miner Res 1994; 9 (1 Suppl):S178.

18. Royal $\mathrm{H}, \mathrm{McNeill} \mathrm{BJ}$. The evaluation and impact of diagnostic test. In: Gottschalk A, Hoffer PB, Potchen EJ, editors. Diagnostik nuclear medicine. 2nd ed. Baltimore: Williams and Wilkins, 1978:15-30.

19. Swets JA. Measuring the accuracy of diagnostic systems. Science $1988 ; 240: 1285-93$.

20. Parfitt AM. Bone remodeling: relationship to the amount and structure of bone, and the pathogenesis and prevention of fractures. In: Riggs BL, Melton LJ, editors. Osteoporosis. New York: Raven Press, 1988:45-96.

21. Uebelhart D, Schlemmer A, Johansen JS, Ginetys E, Christiansen C, Delmas PD. Effect of menopause and hormone replacement therapy on urinary excretion of pyridinium cross-links. J Clin Endocrinol Metab 1991; 72:367-73.

22. Gertz BJ, Shao P, Hanson DA, Quan H, Harris ST, Genant HK, Chesnut CH III, Eyre DR. Monitoring bone resorption in early postmenopausal women by an immunoassay for crosslinked collagen peptides in urine. J Bone Miner Res 1994; 9:135-42.

23. Garnero P, Shih WJ, Ginetys E, Karpf DB, Delmas PD. Comparison of new biochemical markers of bone turnover in late postmenopausal osteoporotic women in response to alendronate treatment. J Clin Endocrinol Metab 1994 c; 79:1693-700.

24. Body JJ, Delmas PD. Urinary pyridinium cross-links as markers of bone resorption in tumor-associated hypercalcemia. J Clin Endocrinol Metab 1992; 74:471-75.

25. Beardsworth LJ, Eyre DR, Dickson IR. Changes with age in the urinary excretion of lysyl- and hydroxylysylpyridinoline, two new markers of bone collagen turnover. J Bone Mineral Res 1990; 5:671-6.

\section{Received July 4/October 7, 1996}

Corresponding author: Paolo Bettica, MD, PhD, Servizio di Endicrinologia, Ospedale "L. Sacco", Via G. B. Grassi 74, I-20257 Milano, Italy 